\title{
An Enhanced Routing and Lifetime Performance for Industrial Wireless Sensor Networks
}

\author{
J. V. Anchitaalagammai ${ }^{1, *}$, K. Muthumayil ${ }^{2}$, D. Kamalraj Subramaniam ${ }^{3}$, Rajesh Verma ${ }^{4}$, \\ P. Muralikrishnan ${ }^{5}$ and G. Visalaxi ${ }^{6}$
}

\footnotetext{
${ }^{1}$ Department of Computer Science and Engineering, Velammal College Engineering and Technology, Madurai, 625009, India

${ }^{2}$ Department of IT, PSNA College of Engineering and Technology, Dindigul, 624622, India

${ }^{3}$ Department of ECE, Karpagam Academy of Higher Education, Coimbatore, India

${ }^{4}$ Electrical Engineering Department, King Khalid University, Abha, 62529, Saudi Arabia

${ }^{5}$ Department of ECE, K.Ramakrishnan College of Engineering, Tiruchirappalli, 621112, India

${ }^{6}$ Department of Computer Science and Engineering, Bharath Institute of Higher Education and Research, Chennai, 600073

*Corresponding Author: J. V. Anchitaalagammai. Email: anchitaalagammailphd@gmail.com

Received: 15 June 2021; Accepted: 16 July 2021
}

\begin{abstract}
Industrial Wireless Sensor Networks (IWSNs), especially energy resources, are scarce. Since sensor nodes are usually very dense, and the data sampled by the sensor nodes have high redundancy, data aggregation saves energy, reduces the number of transmissions, and eliminates redundancy. Many applications can be used in IIWSNs, and a new technique is introduced to detect multiple sensors embedded in different sensor nodes. Packets created by different applications have different properties. Sensors are resource-constrained devices because it is necessary to find effective reaction analysis methods and transfer sensed data to base stations. Since sensors are resource-constrained devices, efficient topologies require data distribution from base stations for management, aggregation, and cloud services in the business tier. To propose a Distributed time series Convergence Routing Protocol (DTSCR) is introduced to detect the correct relay node from the source sensors and transmit the data with the data aggregation node help of the eDCP (Edge Data Collection Protocol). The node will send data to the target source only when those in the process transmit the data, and the cutting edge of the terminal generates a trusted merger. This method can provide a reliable and efficient routing path scheme for information collection and dissemination with maximized packets exchanged between nodes and their Neighbor. Recognizes a specific set of sensor network applications to be flexible to this measurable range. We are also improving the DTSR roaming with both network size and node density.
\end{abstract}

Keywords: Distributed time series routing protocol; edge data collection protocol; industrial WSN

\section{Introduction}

Wireless sensor networks are used in a variety of fields, from environmental monitoring to sniper localization. Wireless components' easy installation and reduced installation costs are the main reasons to

This work is licensed under a Creative Commons Attribution 4.0 International License, which permits unrestricted use, distribution, and reproduction in any medium, provided the original work is properly cited. 
consider wireless solutions for industrial applications. This has led to the increased popularity in the industry, especially for companies interested in automating industrial processes. Wireless Sensor Network (WSN) is a low-power wireless fine dust deep, low-power wireless dust detection with high-resolution CPU and memory, and networking system with environment and major partner network strategies. The advantage of wireless sensor networks is their flexibility and scalability. It is self-contained, and the wireless communication capacity characteristics allow sensor nodes to be deployed in WSN mode without the existing infrastructure in remote and hazardous locations. Wireless sensor nodes communicate directly with other nodes in their vicinity and reach remote nodes via multi-hop communication. It has very limited resources with limited power, bandwidth, processing power, storage,and computing power. Therefore, when a fault occurs, the sensor node is mainly inoperable and irreplaceable due to energy consumption. Improving the sustainable development and longevity of the network is a key issue in modern research sensors. Power consumption is usually dominated by advanced wireless transmission. The exhaustion of radio communication energy is directly related to any transmission on the network. Reduce wireless transmission and increase the number of life-cycle clustering technologies for sensor networks. As a result, the technology effectively coordinated pots, various sensor applications, robot control, environmental control, offices, smart homes, the production environment, the human body communication, and sensor networks are added, such as underwater lived. The main source of energy use is data communication modules. Transmission and acquisition controls that devote part of the information consumption measure more energy. Power usage measurement is mainly two basic parameters; the amount of information sent, the communication range is used for the transmission. Conversely, suppose the increase in the amount of information is an additional increment over the cost of transmitting the information. It is used for communication to minimize the information, so the communication cost is also reduced. In the rest of the paper, Section 2 describes the related work, Section 3 describes the proposed Equations and Mathematical Expressions, Section 4 describes the result and discussion. Finally, Section 5 describes the conclusion.

\section{Related Work}

Wireless sensor networks to detect indoor and industrial environments, avoid obstacles with obstacles and include three-dimensional rangefinders for moving static and dynamic obstacle micro-flying robots. It is only necessary to track the controller of the micro-flying robot. There are no complicated calculations for micro-flying robots in the path [1]. But for industrial wireless sensor networks, it can guarantee the reliability, so it is a useful task in low-power communication and harsh wireless environments. The first QoS framework [2] provides a hybrid wired/wireless network that guarantees that any waiting time can be combined and target reliability for each application.

The central fusion method requires communication and high-bandwidth computing of the processor fast and is not robust due to a single failure point. The identification in this document is to estimate the parameters of the space-time Volterra model in the network using the data distribution system to remove these limitations. A large amount of data can be processed by assigning FC processing tasks to wireless sensor network nodes [3]. Prototypes made from industrial production workshops provide high-reliability and reliable wireless sensing with low application failure rates for measurement, thus providing more confidence for industrial automation to realize what you can do during operation [4]. Based on these characteristics, the risk analysis of industrial activities is based on real-time algorithms, and a large amount of data is collected from within the wireless sensor network. The algorithm allows the sensor node to the screen to receive signal strength indications and remaining energy information based on the established risk analysis in this clustering data transmission structure based on the data collected by the environment or equipment [5]. 
An optimal delay control scheme based on the channel's transfer delay can reduce the total time spent on the sensor nodes by solving the common wireless channel function. Specifically, channel degradation has been described by examining the probability density function of the received signal level across the speed of the first industrial radio environment [6]. The key hybrid industrial system presents a comprehensive demand/supply function analysis based on its first organizational method. Also, consider routing and routing graphical signal sources. If the system is in low critical mode, first, the source route considers each flow schedule in [7]. Clustering and routing are then grouped for a detailed analysis of the relationship between large wireless sensor networks used for routing protocols for reliable and efficient data collection. The node is connected to the maximum transmission distance constraint [8] and uses a route backoff timer and gradient to produce an effective inter-cluster topology. However, the formation of clusters is the overhead of the cluster head in this algorithm's process. To solve this problem, wireless sensor networks, many researchers have come up with the idea to make decisions using fuzzy logic. These guidelines [9] can improve the network lifetime, and to balance the load on the sensor nodes can be adaptively $\mathrm{CH}$ flexible.

An improved hierarchical clustering method has been proposed to achieve wireless sensor networks' energy efficiency using overlapping and adjacent nodes in the sleep-wake mechanism. This minimizes data redundancy, which in turn maximizes network life. Compared to previous hierarchical routing protocols, which require all nodes to collect and send data, this method only occurs when the energy consumption WSN critically performs these tasks [10]. The priority table is formed by prioritizing the two shortest paths to $\mathrm{CH}$ or sink according to some simple and efficient rules. The rule is to use some routing metrics, such as range/power and remaining energy. Simulation results show that the proposed routing protocol can also be integrated with more WSN [11] clustering algorithms. The cluster head $(\mathrm{CH})$ is selected based on energy, reliability, signal-to-interference plus noise ratio, and load residual parameters. Intra-cluster routing is then done using a probabilistic routing method based on network coding. A fuzzybased clustering protocol helps determine the participating cooperative nodes (CNs) in a cluster [12]. The condition for the cluster formation is that d-CPCCA does not fail to address the main components in any cluster. SODCC is extensible and has the same or more complexity than other well-known SOA (Selforganizing algorithms) information [13].

A new method for analyzing regional energy awareness clustering using individual nodes and regional energy awareness clustering is wireless network energy. The head of the cluster is selected based on weights. The weight is determined based on each sensor's remaining energy and the average energy of all sensors in each group. Improperly designed distributed pool algorithms will continue to separate nodes from the channel. Such separated nodes communicate through excessive power consumption [14] sinks. The attenuation model and the chamber model describe changes in the signal power from the propagation distance and the opportunity. To minimize energy consumption, the Taylor series expansion in the compartment model [15] is used to calculate the optimal number of clusters for different orders.

\section{Equations and Mathematical Expressions}

The proposed Distributed Time Series Convergence Routing Protocol (DTSCR) low packet transmission, while establishing the connection will be provided with the effective data collection is implemented with the intention of WSN. This industrial wireless sensor network collects sensing data to send to base stations from the industrial environment with sink nodes' help. Trust the collected sensor node data to send Edge Data Collection Protocol (EDCP) help. This proposal is made at WSN by improving the aggregation rate by forming an intra-network, efficient data delivery to DTSCR. The proposed implementation of link quality is based on low packet loss, transmission speed, throughput performance. 
The proposed method block diagram is shown in Fig. 1. The problem associated with the maximum number of shortest paths has not been considered to solve the network by using Bidirectional Topological Sort (DTS) to improve the throughput and reduce the energy consumption of data collection, and the network improves the life.

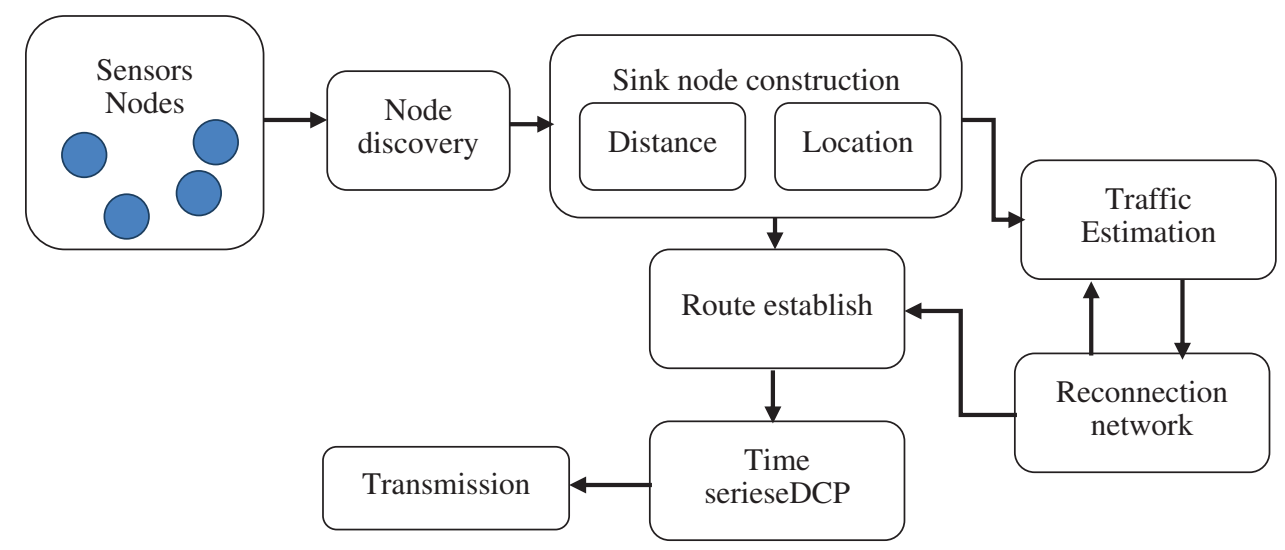

Figure 1: Proposed method block diagram

\subsection{Edge Sink Node Construction}

When the sensor collects data from different corners of the tank is to WSN. Only energy resources decreased absorption path length. Therefore, it isn't easy to gain access to the network to reach all the sensor nodes. The mobile receiving systems search to meet the navigator's controls in a specific way and create an environment with the maximum number of nodes at the entrance edge. A collection point (CP) is considered to be each cluster at any physical location within it. The central point of each sink in DEPC is assumed to be the CP of the sink node. After this, data collection will be completed through this CPS to improve the network's life cycle.

\section{Algorithm steps}

Step 1: Topological sort (Vertex $l$, node $n$ ) and graph $g$

Location $n(x, y)$ where the node positions $x$ as a vertical and $y$ is horizontal in the network.

Step 2: identify the node edge point add it to the routing table as

For ( $i=$ l.start) $; i !=l$. end $;++)$

If (l.visit[n(i)])

$\operatorname{Add}(l, g), \operatorname{stack}(l)$.

End

For $(i=0 ; i<l ; i++)$

If (visit $(n)==$ false)

$\operatorname{radd}(n(i))$

End

Step 3: Finally, unitized silk node and sorted node in network added to the routing table.

After this, the sensor node responds with the requested data along the original path. In this process, to achieve in eDCP using a bidirectional Topological Sort algorithm to find the best path and collect the data each sink in an industrial network. 


\subsection{Traffic and Reconnection}

The proposed TRSP method is suitable for reconnecting network structures separated from their larger sensor element groups. By using this network structure to connect, efficient data collection is completed from the sensor network. The data collection process is shows in Fig. 2. It is placed at the separation node's exact location to realize the reconfiguration process of the transportation network structure. In this method, the distance measurement of the sink node and the center average point analysis method are split into two parts, as shown in Fig. 3. First, the gap of affected nodes in transportation networks was measured using time-based traffic estimation. Let us consider the edge of the sensor network as 'I and node position vertex as 'the integers value points are ' $\mathrm{X}$ ' and ' $\mathrm{Y}$ '.

Sink node position $=\sum_{i=1}^{e} \operatorname{Dir}(v(i))$

\footnotetext{
Algorithm steps

Step 1 initialize the network node vertex as $v$ and node as $n$ and variable $x, y$.

Step 2: identify the node position in the network area using Eq. (1).

Step 3: compute the distance point into the sink node

distances $=\sum_{i=1, j=i+1}^{n}$ dis $($ root,$n(i, j))$

Step 4: participate node list add to the routing table following Edge sink node construction algorithm steps.

Step 5: establish the center point on the network and route between the two nodes.
}

The sensor network is separated from the network by multiple sensor nodes whose nodes do not participate in the network operation partition. Where Eq. (1) estimates the sink node direction and position in the network.

\subsection{Time Series Edge Data Collection}

The sensors in a particular cluster node are time series adhesives. Therefore, it is very likely that collecting and sharing data about one's own future commemorative events are the same. Spatially correlated observation measurements are used to provide WSN time-series data at intervals between sensor nodes. Let us consider the nodes, N1, N2, N3, where the sensor records similar time-series observations in the past.

$b \rightarrow \operatorname{collection}(\operatorname{time}(n 1, n 2, n 3))$

The data collection process is shown in Fig. 4 from Eq. (3), the sensors are N1 and N2, and the observation (b) is based on $\mathrm{N} 3$, the nodes observed in the same time series.

\footnotetext{
Algorithm steps

Begin initialize the network nodes and sink node sn

For each $n$ to sn

Estimate the collection observation time using Eq. (3).

The observation data transmit to the Access point of the base station.

The actives are store in the routing table.

End
} 
In this method group, a dynamic sensor node uses this time correlation. A node in the sensor's immersion step also has a strong chronological correlation similar to observations.

\section{Result and Discussion}

The purpose of our simulation is to observe the appearance of wireless networks. The iterative environment is designed using NS-2 (C++ language algorithm) and is used as a semantic tool for command languages. It leads the allowance for the Advanced Tools Command Language (TCL). The proposed method is performed using a simulation of all networks, after which all code is written into a TCL script. According to the simulation result, the given output simulation result is taken.

Table 1: Simulation parameters of the proposed method

\begin{tabular}{ll}
\hline Parameter & Value \\
\hline Number of nodes & 100 \\
Packet size & $512 \mathrm{~kb}$ \\
Data size & $40 \mathrm{mb}$ \\
Number of packets & 80 \\
Routing protocol & DTSCR, AODV \\
\hline
\end{tabular}

Above Tab. 1 this shows that it is an IoT cloud performance evaluation using the simulation parameters of the proposed method. This section analyzes the routing performance, throughput analysis and time complexity of data packets, which we are discussing.

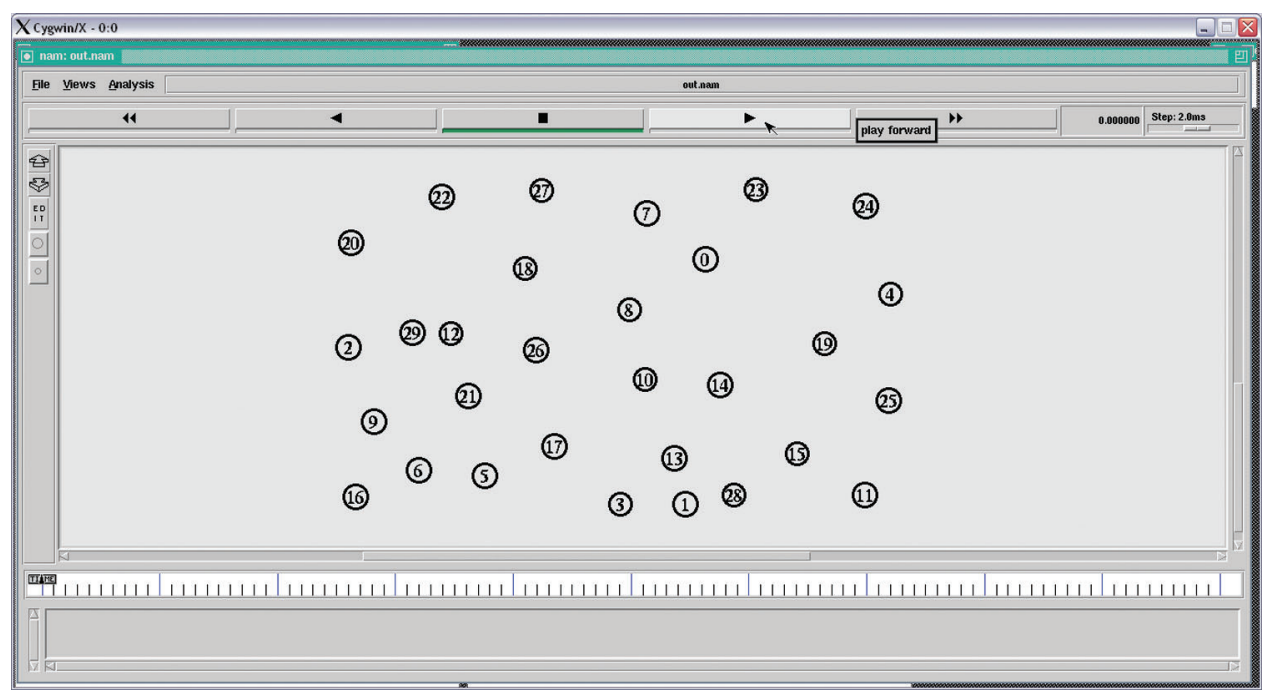

Figure 2: Network initialization process

These industrial sensor node points are shown in Fig. 3 for the process with the network. It node is created in windows with the help of geometrical $\mathrm{x}$ and $\mathrm{y}$-axis points. 


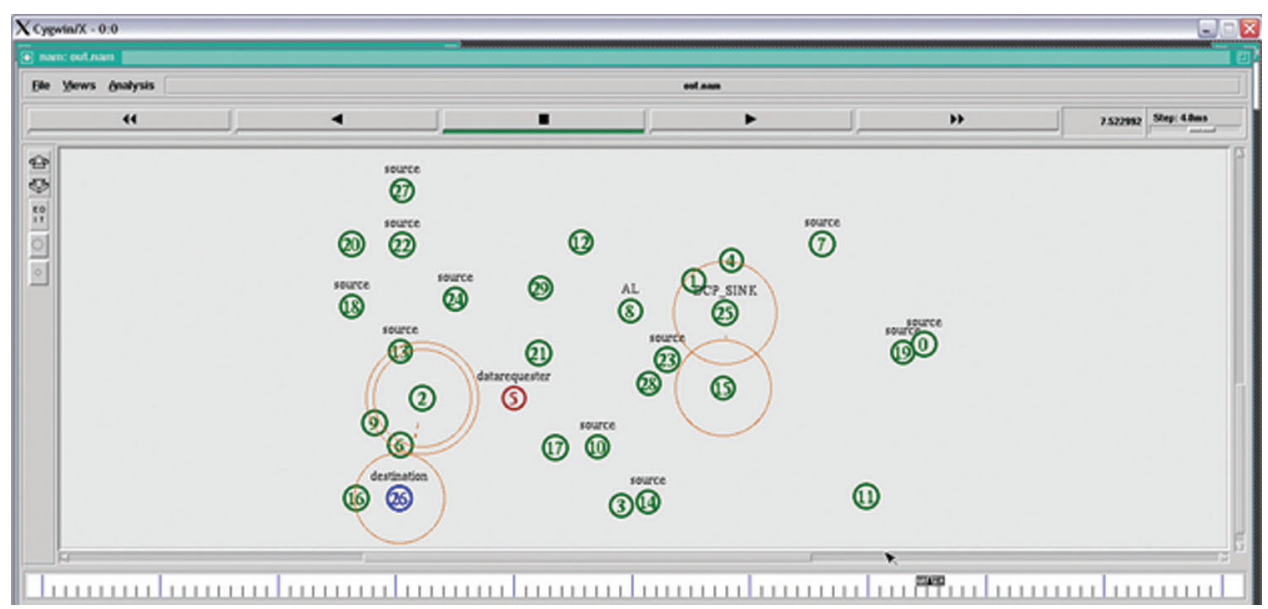

Figure 3: Data collection process

Fig. 3 shows the collecting of the data from sink nodes with the help of DCP sinks. The proposed method Distributed time series Convergence Routing Protocol (DTSCR) compare to the existing method Distributed Graph Routing Algorithm (DGR) and Q-learning reliable routing (QLRR) are analyzed.

packet delivery ratio $=\frac{\text { number of the packet received }}{\text { several packet send }}$

According to the analysis in Fig. 4, the proposed method DTSCR is compared with the existing method QLRR and the data packet transfer ratio DGR. The results show that compared with the existing method DGR, this method has a $93.4 \%$ higher data packet transfer rate than $85.7 \%$. Data packet delivery. Provide $91.2 \%$.

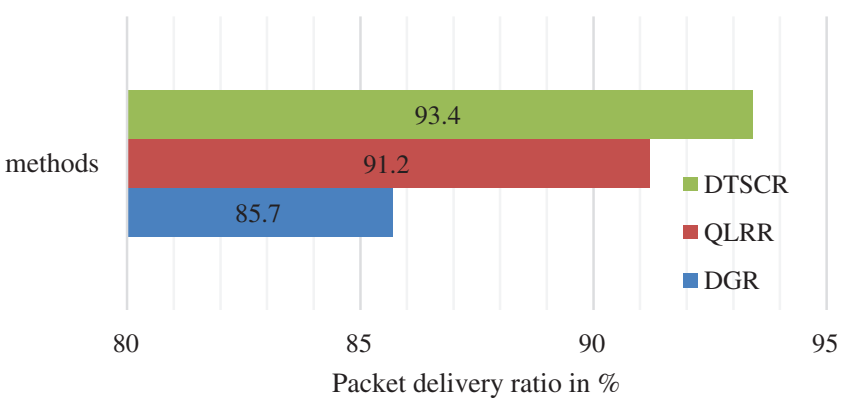

Figure 4: Average packet delivery ratio

This is to obtain the total amount of data received in the last packet, defined as the source from the destination packet divided by 1000 . Throughput is the number of bits transferred per second.

Throughput packetsize $*$ recv.packet $* 8.0 / 1000$

Fig. 5 shows the experimental results of the comparison of Throughput Analysis of existing method DGR, QLRR, proposed method DTSCR, against the $1800 \mathrm{kbps}$ of $200 \mathrm{~s}$. Similarly, the existing method, DGR, QLRR method, has 845 kbps, 934 kbps for $200 \mathrm{~s}$. 
End to End Delay $=\frac{\text { Arrival time }- \text { Sent time }}{\text { Total number of connections }}$

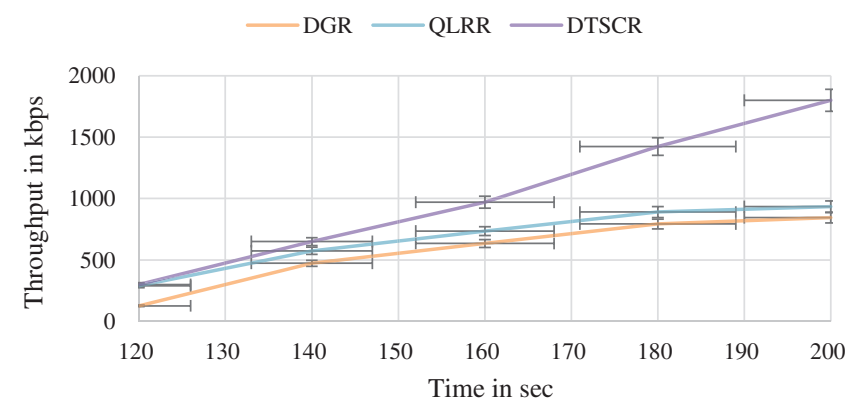

Figure 5: Comparison of throughput analysis

Fig. 6 shows the comparison of the proposed method and the existing method of time complexity taken different nodes like 30,60, 100 nodes. The proposed method provides less time complexity compared to other existing methods. The proposed method DTSCR has $224 \mathrm{~ms}$ of less time complexity compared to the existing method QLRR and DGR.

Routing packet $=\frac{\text { total number of data packets received }}{\text { the total number of routing packets received }} * 100$

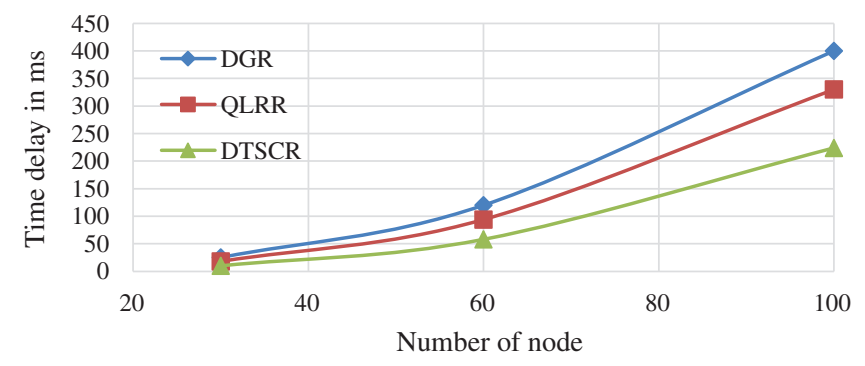

Figure 6: Comparison of End to the End time delay

In this comparison of the proposed method DTSCR and the existing method, QLRR and DGR routing packets are shown Fig. 7. In this analysis of the proposed DTSCR method, results provide $92 \%$ for $200 \mathrm{~s}$, and the existing method has $71 \%, 64 \%$, and $30 \%$ for 200,180 , and $160 \mathrm{~s}$ of rate.

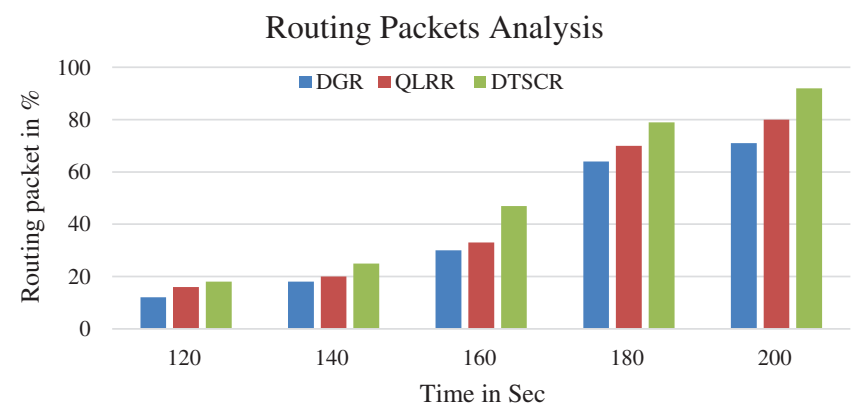

Figure 7: Comparison analysis of routing packets 


\section{Conclusion}

This research aims to the transmission time of each sensor deployed in the network to find the timeconsuming selection of the appropriate sensor nodes to improve the life cycle of the sensor network. Within the surveillance area, scatter sensors have two ways to send observations to a receiver, either single or multi-hop. When multi-hop communication is adopted, the nearest sink node is ejected early. There can be holes near the sink node for heavy traffic because its packet demand is higher than other nodes - this issue overcome to use the DTSCR method to reduce data communication and higher routing packet. The eDCP performs collected data on each edge node. It reduces time delay communication between the nodes if any path or new node enters the network, the re-establish network using Topological Sort (DTS) algorithm. The proposed method DTSCR $93.4 \%$ of packet delivery ratio, $92 \%$ of routing packets, and $224 \mathrm{~ms}$ time delay compared to the existing method. The proposed algorithm provides higher efficiency of throughput performance, lower packet loss, end-to-end time delay, and packet delivery rate than previous methods.

Funding Statement: The authors extend their appreciation to the Deanship of Scientific Research at King Khalid University, Kingdom of Saudi Arabia for funding this work through General Research Project under the Grant Number (RGP. 1/262/42).

Conflicts of Interest: The authors declare they have no known competing financial interests or personal relationships that could influence the work reported in this paper.

\section{References}

[1] H. Li and A. V. Savkin, "Wireless sensor network-based navigation of micro flying robots in the industrial internet of things," IEEE Transactions on Industrial Informatics, vol. 14, no. 8, pp. 3524-3533, 2018.

[2] S. Zoppi, A. Van Bemten, H. M. Gursu, M. Vilgelm, J. Guck et al., "Achieving hybrid wired/wireless industrial networks with wDetServ: Reliability-based scheduling for delay guarantees," IEEE Transactions on Industrial Informatics, vol. 14, no. 5, pp. 2307-2319, 2018.

[3] S. Gupta, A. K. Sahoo and U. K. Sahoo, "Wireless sensor network-based distributed approach to identify Spatiotemporal volterra model for industrial distributed parameter systems," IEEE Transactions on Industrial Informatics, vol. 16, no. 12, pp. 7671-7681, 2020.

[4] K. Yu, M. Gidlund, J. Akerberg and M. Bjorkman, "Performance evaluations and measurements of the realflow routing protocol in industrial wireless networks," IEEE Transactions on Industrial Informatics, vol. 13, no. 3, pp. 1410-1420, 2017.

[5] X. Ding, X. Tian and Y. Yu, "A real-time big data gathering algorithm based on indoor wireless sensor networks for risk analysis of industrial operations," IEEE Transactions on Industrial Informatics, vol. 12, no. 3, pp. 12321242, 2016.

[6] Q. Li, N. Zhang, M. Cheffena and X. Sherman, "Channel-based optimal backoff delay control in delayconstrained industrial wsns," IEEE Transactions on Wireless Communications, vol. 17, no. 12, pp. 1-15, 2019.

[7] C. Xia, X. Jin, L. Kong and P. Zeng, "Bounding the demand for mixed-criticality industrial wireless sensor networks," IEEE Access, vol. 5, pp. 7505-7516, 2017.

[8] H. Haifeng, W. Xiaodong, Y. Zhen and Z. Baoyu, "A spectral clustering approach to identifying cuts in wireless sensor networks," IEEE Sensors Journal, vol. 15, no. 3, pp. 1838-1848, 2015.

[9] X. Zhezhuang, C. Liquan, C. Cailian and G. Xinping, "Joint clustering and routing design for reliable and efficient data collection in large-scale wireless sensor networks," IEEE Internet of Things Journal, vol. 3, no. 4, pp. 520-532, 2016.

[10] S. M. Hosseini and M. H. Kahaei, "Target detection in cluster-based WSN with massive MIMO systems," Electronics Letters, vol. 53, no. 1, pp. 50-52, 2017.

[11] E. Vishnupriya, T. Jayasankar and P. MaheswaraVenkatesh, "SDAOR: Secure Data Transmission of Optimum Routing protocol in wireless sensor networks for surveillance applications," ARPN Journal of Engineering and Applied Sciences, vol. 10, no. 16, pp. 6917-6931, 2015. 
[12] M. Farsi, M. Badawy, M. Moustafa, H. Arafat Ali and Y. Abdulazeem, "A congestion-aware clustering and routing (CCR) protocol for mitigating congestion in WSN," IEEE Access, vol. 7, pp. 105402-105419, 2019.

[13] S. Lata, S. Mehfuz, S. Urooj and F. Alrowais, "Fuzzy clustering algorithm for enhancing reliability and network lifetime of wireless sensor networks," IEEE Access, vol. 8, no. 3, pp. 66013-66024, 2020.

[14] P. Neamatollahi, M. Naghibzadeh, S. Abrishami and M. Yaghmaee, "Distributed clustering-task scheduling for wireless sensor networks using dynamic, hyper round policy," IEEE Transactions on Mobile Computing, vol. 17, no. 2, pp. 334-347, 2018.

[15] J. Qiao and X. Zhang, "Compressive data gathering based on even clustering for wireless sensor networks," IEEE Access, vol. 6, pp. 24391-24410, 2018. 